\title{
Prevalence and risk factors associated with tobacco smoking among adults in India: a nationally representative household
}

\section{survey}

\author{
BENOJIR AHAMMED ${ }^{1, A-F}$, MD. MANIRUZZAMAN ${ }^{1, C-E}$, SUBARNA KUNDU ${ }^{1, c}$, \\ ORCID ID: 0000-0001-8208-9579 \\ JUBAYER AL MAHMUD ${ }^{1,}$, FARZANA FERDAUSI', E-F
}

${ }^{1}$ Statistics Discipline, Khulna University, Khulna, Bangladesh

${ }^{2}$ Khulna Medical College, Khulna, Bangladesh

A - Study Design, B - Data Collection, C - Statistical Analysis, D - Data Interpretation, E - Manuscript Preparation, F - Literature Search, G - Funds Collection

Summary Background. Tobacco smoking is a global epidemic among adults and increases the risk of different diseases, and premature deaths.

Objectives. This study attempts to investigate and estimate the prevalence and risk factors of tobacco smoking among adults in India. Material and methods. This study utilized cross-sectional data from the nationally representative 2017 Global Adult Tobacco Survey. Chi-square analysis was used to show the association between tobacco smoking and risk factors. Binary logistic regression was used to determine the risk factors of tobacco smoking based on $p$-values and odds ratio along with confidence interval.

Results. The overall prevalence of tobacco smoking was $12.84 \%$ (Male: $25.01 \%$, Female: $2.63 \%$ ). The highest prevalence of tobacco smoking was in Meghalaya (35.04\%) and the lowest in Goa (3.07\%). Genderwise, the highest prevalence of tobacco smoking was $57.27 \%$ in Meghalaya for males and $7.27 \%$ in Manipur for females. This study found that 16 out of 19 covariates were significantly $(p<0.001)$ associated with tobacco smoking. Age, region, gender, education, religion, marital status, wealth index, number of living persons, and presence of smoking persons were found to be highly significant $(p<0.001)$ risk factors. Adults from $46-60$ years, north east region, no education, daily wage/casual labourers and self-employed, non-Hindu, married. Poorest, presence of smoking persons, and no knowledge about smoking cause strokes had significantly higher risk of tobacco smoking.

Conclusions. Tobacco smoking remains one of the major causes of diseases, deaths and economic losses. This recent realistic evidence will help policy makers to make policy for reducing tobacco smoking in India, as well as different states.

Key words: disease, logistic models, prevalence, risk factors, tobacco smoking.

Ahammed B, Maniruzzaman Md., Kundu S, Al Mahmud J, Ferdausi F. Prevalence and risk factors associated with tobacco smoking among adults in India: a nationally representative household survey. Fam Med Prim Care Rev 2019; 21(4): 307-317, doi: https://doi. org/10.5114/fmpcr.2019.86505.

\section{Background}

Tobacco smoking is the common practice of smoking in India. Tobacco smoking is one of the major public health threats in the world [1], and tobacco is a highly addictive material [2]. Globally, tobacco smoking remains one of the most important causes of diseases and deaths [3-5]. Two third of the tobacco smokers in the world live in low and middle income countries [6]. India has one of the top tobacco users in the world, and one of the fewer countries in the world where the prevalence of tobacco smoking is high [7]. In 1998-1999, the national family health survey second round found that the prevalence of tobacco use in India was approximately $37 \%$ among adults of aged 15 years and above [8]. The problem of tobacco use is significantly concomitant with a high mortality problem. The number of tobacco users is increasing all over the world, and the global prevalence of tobacco smoking among adults is $22 \%$ [9]. Tobacco smoking is one of the leading causes of various preventable diseases and premature deaths. In India a large number of premature deaths occur through tobacco smoking, and the majority of smoking related deaths occur in the prime working age group of under 60 years [10]. According to the World Health Organization (WHO), approximately six million deaths occur every single year due to tobacco smoking, and in 2030 these deaths will be more than eight million per year [11]. A global adult tobacco survey was conducted among three billion individuals from 16 countries, and the survey showed that approximately $48.6 \%$ of males and $11.3 \%$ of females were tobacco users [12]. A recent study found that worldwide about 400 million adult deaths will occur through tobacco smoking between the year 2010 and 2050, and most of the deaths will occur in the age group 30-69 years [13].

Another burden of tobacco smoking is the economic costs. Tobacco smoking costs can be categorized into direct, and indirect costs, and around $15 \%$ of the aggregate health care expenditure in high income countries can be credited to smoking [14]. The expenditure on purchasing tobacco contributes to household poverty [2]. In addition, the treatment costs of tobacco smoking related diseases were higher among tobacco smoking families. All the problems of tobacco smoking are public health and socioeconomic problems. The design and implementation of appropriate policies for controlling tobacco smoking is very important for the improvement of public health disease [2]. So understanding the factors that influence the tobacco smoking status of adults is important to policymakers as well as researchers. India is one of the countries in Asia that shares the burden of tobacco smoking epidemics. The Indian government has taken different steps to reduce the prevalence of tobacco 
smoking. An effective tobacco smoking policy mainly depends on the assessment of the prevalence of tobacco smoking. This is an important step in reducing tobacco use in the country. There are several national level studies that have been conducted to collect information on tobacco use to evaluate the prevalence of tobacco use. Among the national level studies, the National Family Health Survey (NFHS), the National Household Survey of Drug and Alcohol Abuse in India (NHSDAA), and the Global Adult Tobacco Survey (GATS) are notable [7]. All these studies accumulated data on tobacco use as a component of health knowledge, attitudes and perceptions based surveys. Most of the previous research work on tobacco smoking was based on localized studies with sociodemographic predictors of tobacco related behaviour.

\section{Objectives}

This study is an effort to examine the prevalence and regional variations of tobacco smoking among adults in India using large, nationally representative, and more recent Global Adult Tobacco Survey (GATS) data. Therefore, this study also identifies the different associated factors and impacts of tobacco smoking among adults in India.

\section{Material and methods}

\section{Data sources}

The study was based on a nationally representative sample of adults ( $\geq 15$ years of age) living in India, and the data extracted from the 2017 Global Adult Tobacco Survey (GATS-2017) India. The Global Adult Tobacco Survey (GATS), a component of the Global Tobacco Surveillance System (GTSS), is a global standard for systematically monitoring adult tobacco use and tracking key tobacco control indicators. After excluding all unnecessary and missing information of adult respondents, 73,757 adults were finally used in this study.

\section{Sample design}

The multistage stratified cluster sample design was used in GATS-2017 India data. The sample size was 84,047 selected households with 74,037 completed individual interviews. The questionnaire of GATS India included fundamental questions about background characteristics, tobacco smoking, smokeless tobacco, cessation, secondhand smoke, economics, media, and knowledge, attitudes and perceptions. All the questions were reviewed and improved. Finally, questions were approved by a questionnaire expert review committee. GATS is designed to produce national and sub-national estimates among adults across countries. The target population includes all men and women who are 15 years of age or older. All members of the target population were sampled from the household that was their usual place of residence. Data were collected using electronic handheld devices. The survey covered fields like tobacco use (smoking and smokeless tobacco), exposure to second hand smoke, cessation, economics of tobacco, exposure to media messages on tobacco use, and knowledge, attitudes and perceptions towards tobacco use. The survey was designed to provide estimates of tobacco prevalence at national and state levels. Data on tobacco use were collected from eligible respondents aged 15 years and above.

\section{Outcome variable}

In this study the outcome variable is tobacco smoking status. The tobacco smoking status of respondents was determined by answers ("daily", "less than daily", and "not at all") to the question "Do you currently smoke tobacco?" Respondents who answered "not at all" were categorized as "non-smokers", whilst those who answered "daily" or "less than daily" were considered "current smokers".

\section{Independent variables}

The national level and state level variations in tobacco smoking were measured for the 6 national region and 32 states of India. The variation in tobacco smoking was also assessed for 14 age groups. The outcome variables were studied against all selected potential risk variables, and these variables were divided into different groups, namely: sampling variables, household variables, and background characteristics of the respondents, media exposure variable, and knowledge, attitudes \& perceptions of tobacco smoking. Short descriptions of different independent variables along with their categories are given in Table 1.

\begin{tabular}{|c|c|c|}
\hline Variable & Description of variable & Categories of variable for analysis \\
\hline Age (year) & Age of the respondents in group & $15-30$ years, $31-45$ years, $46-60$ years, and $61+$ years \\
\hline Residence & Residence status of respondent & Urban, and rural \\
\hline Region & National region of the respondents & North, Central, East, North East, West, and South \\
\hline Gender & Records the gender of the selected person & Male or female \\
\hline Education & $\begin{array}{l}\text { What is the highest level of education the respondent } \\
\text { has completed? }\end{array}$ & $\begin{array}{l}\text { No education, up to primary, up to secondary, and } \\
\text { more than secondary }\end{array}$ \\
\hline Occupation & $\begin{array}{l}\text { Which of the following best describes your main work } \\
\text { status over the past } 12 \text { months? }\end{array}$ & $\begin{array}{l}\text { Govt/non-govt employee, daily wage/casual labourer, } \\
\text { self-employed, homemaker, student, and others } \\
\text { (retired, unemployed, don't know, refused) }\end{array}$ \\
\hline Religion & What is your religion? & $\begin{array}{l}\text { Hindu, and others (Muslim, Christian, Buddhism, Jain, } \\
\text { Sikh, etc.) }\end{array}$ \\
\hline Marital status & What is your marital status? & $\begin{array}{l}\text { Not married, currently married, and formally married } \\
\text { (separated, divorced, widowed, etc.) }\end{array}$ \\
\hline Wealth index & $\begin{array}{l}\text { This variable is computed using household facilities } \\
\text { and products such as electricity, flush toilet, tele- } \\
\text { phone, mobile phone, television, radio, refrigerator, } \\
\text { car, moped, scooter or motorcycle, washing machine, } \\
\text { computer or laptop, air conditioner, and electric fan }\end{array}$ & Richest, rich, middle, poor, and poorest \\
\hline No of living persons & In total how many persons live in the household? & $1-3,4-6$, and $7+$ \\
\hline
\end{tabular}




\begin{tabular}{|l|l|l|}
\hline \multicolumn{3}{|l|}{ Table 1. Short description of different variables with their categories } \\
\hline Variable & Description of variable & Categories of variable for analysis \\
\hline $\begin{array}{l}\text { Presence of smoking } \\
\text { person }\end{array}$ & $\begin{array}{l}\text { This variable is computed from the smoking history of } \\
\text { different persons in a household }\end{array}$ & Yes or no \\
\hline $\begin{array}{l}\text { Use of smokeless } \\
\text { tobacco }\end{array}$ & $\begin{array}{l}\text { Does the respondent currently use smokeless tobacco, } \\
\text { including betel quid with tobacco, sada or surti, khaini } \\
\text { or tobacco lime mixture, gutkha? }\end{array}$ & Yes or no \\
\hline $\begin{array}{l}\text { Mass media expo- } \\
\text { sure }\end{array}$ & $\begin{array}{l}\text { In the last 30 days have you noticed information about } \\
\text { the dangers of smoking tobacco, or that encourages } \\
\text { quitting in newspapers or in magazines? }\end{array}$ & Yes or no \\
\hline Print media exposure & $\begin{array}{l}\text { In the last 30 days, have you noticed information } \\
\text { about the dangers of smoking tobacco, or that encour- } \\
\text { ages quitting on television or radio? }\end{array}$ & Yes or no \\
\hline $\begin{array}{l}\text { Smoking causes seri- } \\
\text { ous illness }\end{array}$ & $\begin{array}{l}\text { Do you know or believe that smoking tobacco causes } \\
\text { serious illness? }\end{array}$ & Yes, no, or do not know \\
\hline $\begin{array}{l}\text { Smoking causes } \\
\text { stroke }\end{array}$ & $\begin{array}{l}\text { Do you know or believe that smoking tobacco causes } \\
\text { strokes? }\end{array}$ & Yes, no, or do not know \\
\hline $\begin{array}{l}\text { Smoking causes } \\
\text { heart attack }\end{array}$ & $\begin{array}{l}\text { Do you know or believe that smoking tobacco causes } \\
\text { heart attacks? }\end{array}$ & Yes, no, or do not know \\
\hline $\begin{array}{l}\text { Smoking causes lung } \\
\text { cancer }\end{array}$ & $\begin{array}{l}\text { Do you know or believe that smoking tobacco causes } \\
\text { lung cancer? }\end{array}$ & Yes, no, or do not know \\
\hline Smoking causes TB & $\begin{array}{l}\text { Do you know or believe that smoking tobacco causes } \\
\text { TB? }\end{array}$ & Yes, no, or do not know \\
\hline
\end{tabular}

\section{Statistical analysis}

The dataset was analyzed using different statistical tools and statistical techniques. In the base characteristics table for continuous variables they are expressed as mean ( \pm standard deviation), and categorical variables are expressed as frequency (percentages) (Table 3). Frequency scores were also used to compute the prevalence of tobacco smoking status. These descriptive statistics were used to demonstrate the baseline characteristics of the selected potential risk factors. Different graphical (bar diagram, line diagram, etc.) representations were used to display the prevalence of tobacco smoking. This study also examined the observed and expected value by state and gender to show the differences in the prevalence of captures. To do this analysis, firstly this study ran a binary logistic regression model, except three geographic variables, namely: state, region, and place of residence. Then it computed the predicted probabilities from the binary logistic regression. The predicted probabilities are divided into two groups: if the probability value is less than 0.50 , considered as not tobacco smoking $(0)$, otherwise tobacco smoking (1). By using this result this study obtained the average frequency of tobacco smoking status by statewise and compared it to observed prevalence (Table 2). This is a simple way to quantify what accounts for the massive difference in the different states. Analysis of the determinants of tobacco smoking was done on unweighted data as it gives associations of smoking with different considered independent variables. Bivariate analyses were performed to obtain the prevalence of tobacco smoking for various categories of the selected variables. Pearson chi-square $\left(\chi^{2}\right)$ analysis was used to show the association between tobacco smoking and the selected potential risk factors [15]. Moreover, binary logistic regression was used to determine the high risk factors of tobacco smoking based on odds ratio $(\mathrm{OR})$ and $p$-value $[16,17]$. Statistical significance was defined with $p<0.05$ (significant) and $p<0.01$ (highly significant). SPSS version 23.0 and Ri86 3.5.2 were used for analysis.

\section{Ethical approval}

The world worked together to design and implement GATS, by the Centers for Disease Control and Prevention (CDCP), CDC
Foundation, the Johns Hopkins Bloomberg School of Public Health (JHSPH), Research Triangle Institute International (RTI International), the World Health Organization (WHO), and many countries. The GATS-2017 India survey was approved by the Ministry of Health and Family Welfare, Government of India.

\section{Results}

The data were introduced and analyzed based on descriptive statistics and the logistic regression model. The purposes of the study were to measure the prevalence, and identify the risk factors, of tobacco smoking among adults aged 15 years and older in India. Overall, $12.84 \%$ of adults were found to smoke tobacco. The general perception of the prevalence of tobacco smoking is presented by state in Figure 1. The prevalence of tobacco smoking was higher in Meghalaya (35.04\%) and lower in Goa (3.07\%) compared to other states of India.

The prevalence of tobacco smoking is also presented in Figure 2 based on gender in India by state. Among males, the highest proportion of tobacco smoking was $57.27 \%$ in Meghalaya, and the lowest proportion was $6.75 \%$ in Maharashtra. But in females the results were different: the highest amount of tobacco smoking was $17.14 \%$ in Mizoram, and the lowest amount was $0.07 \%$ in Puducherry.

The 32 states of India were divided into 6 national regions as north, central, east, north east, west, and south. Figure 3 presents the prevalence of tobacco smoking among adults, male and female, in India by national region. Among male and female adults the prevalence of tobacco smoking was maximum in the north east region (Male: $38.48 \%$ and Female: $6.73 \%$ ) and minimum in the west region (Males: $10.91 \%$ and Females: 0.82\%).

The prevalence of tobacco smoking according to males and females by 14 age group gives us the real picture of the hazardous age group of tobacco smoking presented in Figure 4 . The highest risk age group for male adults of tobacco smoking was 55-59 years (34.48\%), and the lowest risk age group was 15-19 years $(6.08 \%)$. Among females, the highest risk age group of tobacco smoking was $75-79$ years $(6.65 \%)$, and the lowest risk age group was $15-19$ years $(0.43 \%)$. 


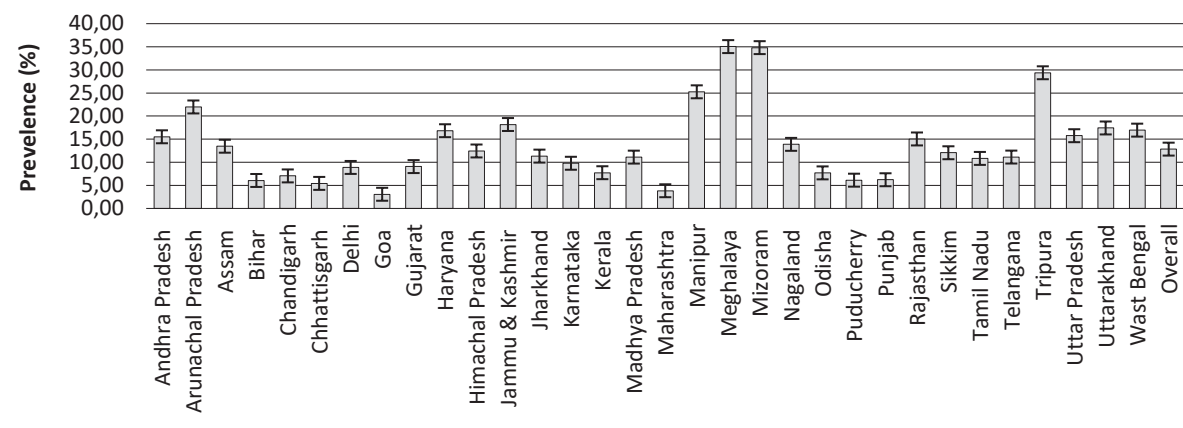

Different States Of India
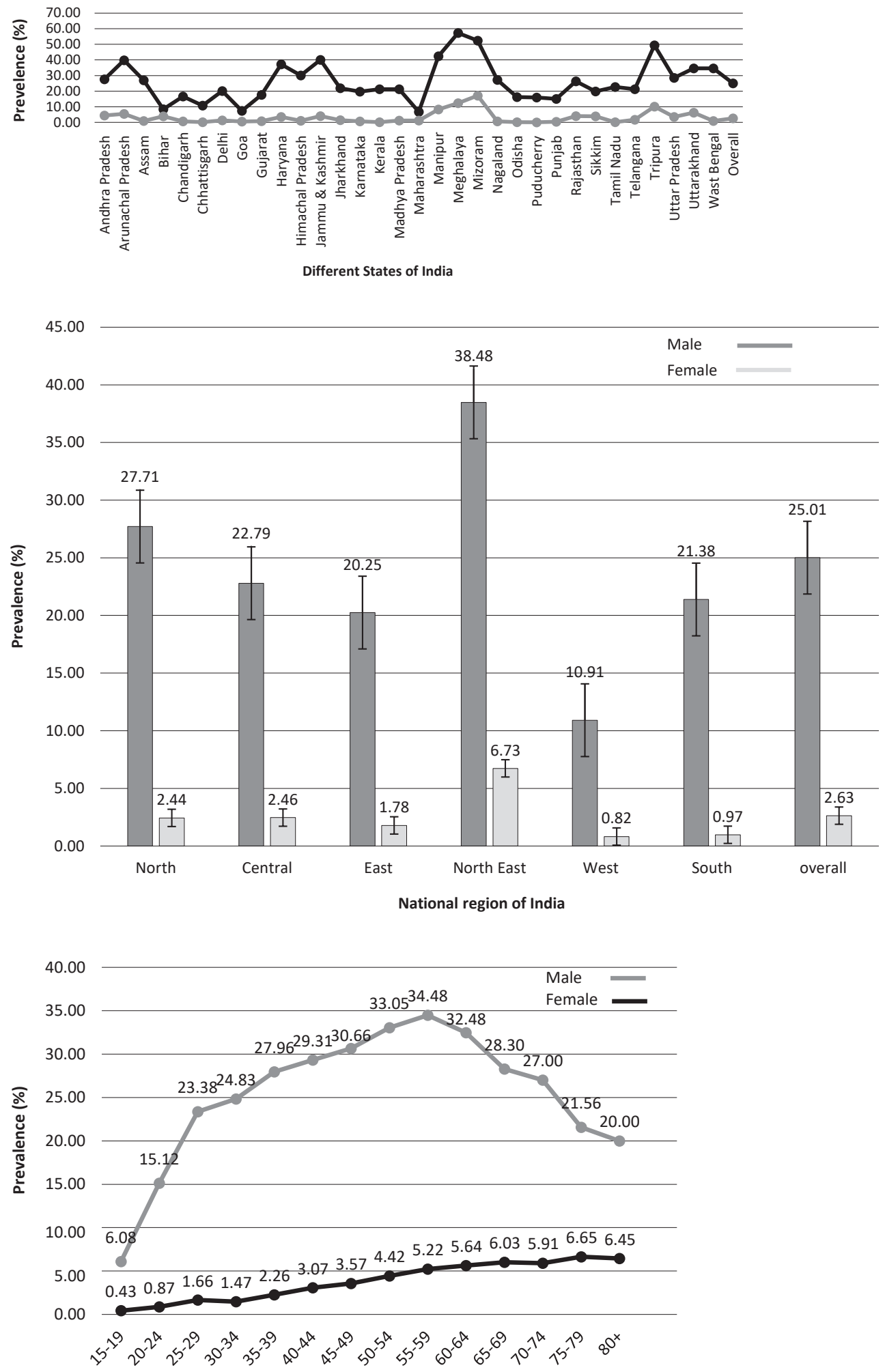

Figure 1. Prevalence of tobacco smoking among adults in India by state

Figure 2. Prevalence of tobacco smoking among adults, male and female, in India by state

Figure 3. Prevalence of tobacco smoking among adults, male and female, in India by national region
Figure 4. Prevalence of tobacco smoking according to gender and age groups in India 
Table 2 presents the observed and expected prevalence of tobacco smoking by state. There was marked state-level variation in the observed prevalence of tobacco smoking, with rates ranging from $3.07 \%$ in Goa to $35.04 \%$ in Meghalaya. The observed prevalence of tobacco smoking was greater than $20 \%$ in Arunachal Pradesh, Manipur, Meghalaya, Mizoram, and Tripura, and between $10 \%$ and $20 \%$ in Andhra Pradesh, Assam, Haryana, Himachal Pradesh, Jammu \& Kashmir, Jharkhand, Madhya Pradesh, Nagaland, Rajasthan, Sikkim, Tamil Nadu, Telangana, Uttar Pradesh, Uttarakhand, and West Bengal.

The estimated prevalence of tobacco smoking for males was between $30 \%$ and 56\% in Andhra Pradesh, Arunachal Pradesh, Haryana, Himachal Pradesh, Jammu \& Kashmir, Manipur, Meghalaya, Mizoram, Rajasthan, Tripura, Uttar Pradesh, Uttarakhand and West Bengal. But for females tobacco smoking was more than $10 \%$ only in Mizoram (15.59\%) state.

Among 73,757 adults only 9,471 smoked tobacco. The overall mean age ( \pm standard deviation) of the respondents was 39.46 ( \pm 15.43 ) years. The mean ( \pm standard deviation) number of family members to every respondent was $4.78( \pm 2.28)$. Table 3 provides the overall summary statistics of the different characteristics of respondents. About $70.49 \%$ of respondents were under 45 years old, and the majority of respondents were female (54.38\%). Around two thirds of the respondents were from rural areas, and the highest $23.17 \%$ lived in the North region in India. The majority of the study population had at least up to secondary education $(30.34 \%)$, and $34.89 \%$ of respondents' were homemakers. About $72.95 \%$ of respondents were Hindu, and $76.99 \%$ were currently married. $41.26 \%$ of respondents were in the poor wealth category, $57.26 \%$ of respondents' family members were in the $4-6$ category. About $82.64 \%$ of respondents' families had no smoking person, and $79.62 \%$ of respondents did not use any smokeless tobacco. $62.20 \%$ of respondents has access to mass media but only $37.18 \%$ had access to print media. The highest portion of the respondents had knowledge about tobacco smoking causing serious illness (92.15\%), strokes (67.69\%), heart attacks (78.53\%), lung cancer (93.68\%) and TB (93.04\%).

\begin{tabular}{|c|c|c|c|c|c|c|c|c|c|}
\hline \multirow[t]{2}{*}{ State } & \multicolumn{3}{|c|}{ Male } & \multicolumn{3}{|c|}{ Female } & \multicolumn{3}{|c|}{ Total } \\
\hline & $n$ & $\begin{array}{l}\text { observed } \\
\text { (\%) }\end{array}$ & $\begin{array}{l}\text { expected } \\
(\%)\end{array}$ & $n$ & $\begin{array}{l}\text { observed } \\
(\%)\end{array}$ & $\begin{array}{l}\text { expected } \\
(\%)\end{array}$ & $n$ & $\begin{array}{l}\text { observed } \\
\text { (\%) }\end{array}$ & $\begin{array}{l}\text { expected } \\
(\%)\end{array}$ \\
\hline Andhra Pradesh & 260 & 27.60 & 30.25 & 45 & 4.39 & 3.32 & 305 & 15.51 & 16.23 \\
\hline $\begin{array}{l}\text { Arunachal } \\
\text { Pradesh }\end{array}$ & 262 & 39.82 & 40.73 & 39 & 5.48 & 4.21 & 301 & 21.97 & 21.75 \\
\hline Assam & 371 & 26.96 & 29.29 & 13 & 0.88 & 0.68 & 384 & 13.47 & 14.49 \\
\hline Bihar & 126 & 8.38 & 11.31 & 62 & 3.87 & 3.68 & 188 & 6.05 & 7.38 \\
\hline Chandigarh & 156 & 16.58 & 17.85 & 9 & 0.64 & 0.21 & 165 & 7.04 & 7.30 \\
\hline Chhattisgarh & 111 & 10.72 & 12.95 & 2 & 0.19 & 0.19 & 113 & 5.42 & 6.53 \\
\hline Delhi & 188 & 20.04 & 22.17 & 17 & 1.24 & 0.73 & 205 & 8.87 & 9.44 \\
\hline Goa & 56 & 7.43 & 8.62 & 6 & 0.47 & 0.16 & 62 & 3.07 & 3.31 \\
\hline Gujarat & 235 & 17.59 & 25.07 & 11 & 0.80 & 1.38 & 246 & 9.06 & 13.04 \\
\hline Haryana & 368 & 37.25 & 39.27 & 53 & 3.50 & 2.44 & 421 & 16.83 & 16.99 \\
\hline Himachal Pradesh & 301 & 30.07 & 31.67 & 15 & 0.97 & 0.65 & 316 & 12.44 & 12.87 \\
\hline Jammu \& Kashmir & 390 & 40.08 & 43.68 & 60 & 3.99 & 3.46 & 450 & 18.17 & 19.26 \\
\hline Jharkhand & 204 & 21.89 & 24.25 & 14 & 1.41 & 1.21 & 218 & 11.33 & 12.37 \\
\hline Karnataka & 254 & 19.66 & 22.45 & 9 & 0.64 & 0.86 & 263 & 9.78 & 11.23 \\
\hline Kerala & 166 & 21.25 & 22.41 & 2 & 0.14 & 0.14 & 168 & 7.73 & 8.15 \\
\hline Madhya Pradesh & 308 & 21.23 & 27.64 & 17 & 1.15 & 0.61 & 325 & 11.11 & 14.02 \\
\hline Maharashtra & 102 & 6.75 & 10.05 & 18 & 1.11 & 0.43 & 120 & 3.83 & 5.08 \\
\hline Manipur & 341 & 42.41 & 42.29 & 67 & 8.25 & 7.27 & 408 & 25.25 & 24.69 \\
\hline Meghalaya & 453 & 57.27 & 55.37 & 96 & 12.37 & 4.12 & 549 & 35.04 & 29.99 \\
\hline Mizoram & 408 & 52.44 & 49.87 & 133 & 17.14 & 15.59 & 541 & 34.81 & 32.75 \\
\hline Nagaland & 216 & 27.27 & 25.76 & 5 & 0.62 & 0.37 & 221 & 13.87 & 12.99 \\
\hline Odisha & 142 & 16.17 & 20.05 & 1 & 0.10 & 0.31 & 143 & 7.70 & 9.63 \\
\hline Puducherry & 150 & 15.89 & 18.11 & 1 & 0.07 & 0 & 151 & 6.12 & 6.93 \\
\hline Punjab & 150 & 15.02 & 17.02 & 6 & 0.40 & 0.4 & 156 & 6.21 & 7.01 \\
\hline Rajasthan & 393 & 26.29 & 30.57 & 62 & 4.05 & 2.55 & 455 & 15.04 & 16.40 \\
\hline Sikkim & 142 & 19.80 & 23.85 & 27 & 3.94 & 3.21 & 169 & 12.05 & 13.77 \\
\hline Tamil Nadu & 311 & 22.70 & 24.60 & 3 & 0.20 & 0.2 & 314 & 10.83 & 11.73 \\
\hline Telangana & 187 & 21.20 & 25.17 & 16 & 1.70 & 1.91 & 203 & 11.12 & 13.15 \\
\hline Tripura & 377 & 49.41 & 51.64 & 80 & 10.09 & 7.44 & 457 & 29.37 & 29.11 \\
\hline Uttar Pradesh & 479 & 28.46 & 30.60 & 62 & 3.54 & 2.45 & 541 & 15.75 & 16.24 \\
\hline Uttarakhand & 328 & 34.56 & 33.19 & 91 & 6.25 & 5.22 & 419 & 17.42 & 16.26 \\
\hline West Bengal & 480 & 34.56 & 37.94 & 14 & 0.92 & 1.05 & 494 & 16.95 & 18.63 \\
\hline
\end{tabular}




\begin{tabular}{|c|c|c|c|c|c|c|c|}
\hline & \multirow{2}{*}{\multicolumn{2}{|c|}{ Total }} & \multicolumn{4}{|c|}{ Tobacco smoking status } & \multirow[t]{3}{*}{$p$} \\
\hline & & & \multicolumn{2}{|l|}{ no } & \multicolumn{2}{|l|}{ yes } & \\
\hline & $n$ & $\%$ & $n$ & $\%$ & $n$ & $\%$ & \\
\hline $\begin{array}{l}\text { Age group } \\
\begin{array}{l}15-30 \text { years } \\
31-45 \text { years } \\
46-60 \text { years } \\
61+\text { years }\end{array}\end{array}$ & $\begin{array}{l}25572 \\
26422 \\
14054 \\
7709\end{array}$ & $\begin{array}{l}34.67 \\
35.82 \\
19.05 \\
10.45\end{array}$ & $\begin{array}{l}23595 \\
22735 \\
11545 \\
6411\end{array}$ & $\begin{array}{l}92.27 \\
86.05 \\
82.15 \\
83.16\end{array}$ & $\begin{array}{l}1977 \\
3687 \\
2509 \\
1298\end{array}$ & $\begin{array}{l}7.73 \\
13.95 \\
17.85 \\
16.84\end{array}$ & $<0.001$ \\
\hline $\begin{array}{l}\text { Residence } \\
\text { Urban } \\
\text { Rural }\end{array}$ & $\begin{array}{l}26414 \\
47343\end{array}$ & $\begin{array}{l}35.81 \\
64.19\end{array}$ & $\begin{array}{l}23901 \\
40385\end{array}$ & $\begin{array}{l}90.49 \\
85.30\end{array}$ & $\begin{array}{l}2513 \\
6958\end{array}$ & $\begin{array}{l}9.51 \\
14.70\end{array}$ & $<0.001$ \\
\hline $\begin{array}{l}\text { Region } \\
\text { North } \\
\text { Central } \\
\text { East } \\
\text { North East } \\
\text { West } \\
\text { South }\end{array}$ & $\begin{array}{l}17088 \\
11470 \\
9801 \\
13509 \\
7870 \\
14019\end{array}$ & $\begin{array}{l}23.17 \\
15.55 \\
13.29 \\
18.32 \\
10.67 \\
19.01\end{array}$ & \begin{tabular}{|l|}
14956 \\
10036 \\
8758 \\
10479 \\
7442 \\
12615
\end{tabular} & $\begin{array}{l}87.52 \\
87.50 \\
89.36 \\
77.57 \\
94.56 \\
89.99\end{array}$ & $\begin{array}{l}2132 \\
1434 \\
1043 \\
3030 \\
428 \\
1404\end{array}$ & $\begin{array}{l}12.48 \\
12.50 \\
10.64 \\
22.43 \\
5.44 \\
10.01\end{array}$ & $<0.001$ \\
\hline $\begin{array}{l}\text { Gender } \\
\text { Male } \\
\text { Female }\end{array}$ & $\begin{array}{l}33647 \\
40110\end{array}$ & $\begin{array}{l}45.62 \\
54.38\end{array}$ & $\begin{array}{ll}25 & 232 \\
39 & 054\end{array}$ & $\begin{array}{l}74.99 \\
97.37\end{array}$ & $\begin{array}{l}8415 \\
1056\end{array}$ & $\begin{array}{l}25.01 \\
2.63\end{array}$ & $<0.001$ \\
\hline $\begin{array}{l}\text { Educational } \\
\text { No education } \\
\text { Up to primary } \\
\text { Up to secondary } \\
\text { More than secondary }\end{array}$ & $\begin{array}{l}18423 \\
16308 \\
22377 \\
16649\end{array}$ & $\begin{array}{l}24.98 \\
22.11 \\
30.34 \\
22.57\end{array}$ & $\begin{array}{l}15674 \\
13409 \\
19703 \\
15500\end{array}$ & $\begin{array}{l}85.08 \\
82.22 \\
88.05 \\
93.10\end{array}$ & $\begin{array}{l}2749 \\
2899 \\
2674 \\
1149\end{array}$ & $\begin{array}{l}14.92 \\
17.78 \\
11.95 \\
6.90\end{array}$ & $<0.001$ \\
\hline $\begin{array}{l}\text { Occupation } \\
\text { Govt./Non-Govt. Employee } \\
\text { Daily Wage/Casual Labourer } \\
\text { Self-Employed } \\
\text { Homemaker } \\
\text { Student } \\
\text { Others }\end{array}$ & $\begin{array}{l}9576 \\
13699 \\
13912 \\
25734 \\
6113 \\
4723\end{array}$ & $\begin{array}{l}12.98 \\
18.57 \\
18.86 \\
34.89 \\
8.29 \\
6.40\end{array}$ & $\begin{array}{l}8230 \\
10485 \\
10769 \\
24977 \\
5940 \\
3885\end{array}$ & $\begin{array}{l}85.94 \\
76.54 \\
77.41 \\
97.06 \\
97.17 \\
82.26\end{array}$ & $\begin{array}{l}1346 \\
3214 \\
3143 \\
757 \\
173 \\
838\end{array}$ & $\begin{array}{l}14.06 \\
23.46 \\
22.59 \\
2.94 \\
2.83 \\
17.74\end{array}$ & $<0.001$ \\
\hline $\begin{array}{c}\text { Religion } \\
\text { Hindu } \\
\text { Others }\end{array}$ & $\begin{array}{l}53804 \\
19953\end{array}$ & $\begin{array}{l}72.95 \\
27.05\end{array}$ & $\begin{array}{l}47591 \\
16695\end{array}$ & $\begin{array}{l}88.45 \\
83.67\end{array}$ & $\begin{array}{l}6213 \\
3258\end{array}$ & $\begin{array}{l}11.55 \\
16.33\end{array}$ & $<0.001$ \\
\hline $\begin{array}{l}\text { Marital status } \\
\text { Not married } \\
\text { Currently married } \\
\text { Formerly married }\end{array}$ & $\begin{array}{l}11906 \\
56782 \\
5069 \\
\end{array}$ & $\begin{array}{l}16.14 \\
76.99 \\
6.87 \\
\end{array}$ & $\begin{array}{l}11028 \\
48670 \\
4588 \\
\end{array}$ & $\begin{array}{l}92.63 \\
85.71 \\
90.51 \\
\end{array}$ & $\begin{array}{l}878 \\
8112 \\
481\end{array}$ & $\begin{array}{l}7.37 \\
14.29 \\
9.49 \\
\end{array}$ & $<0.001$ \\
\hline $\begin{array}{l}\text { Wealth index } \\
\text { Richest } \\
\text { Rich } \\
\text { Middle } \\
\text { Poor } \\
\text { Poorest }\end{array}$ & $\begin{array}{l}3099 \\
12100 \\
18281 \\
30434 \\
9843\end{array}$ & $\begin{array}{l}4.20 \\
16.41 \\
24.79 \\
41.26 \\
13.35\end{array}$ & \begin{tabular}{|l}
2935 \\
11164 \\
16285 \\
25868 \\
8034
\end{tabular} & $\begin{array}{l}94.71 \\
92.26 \\
89.08 \\
85.00 \\
81.62\end{array}$ & $\begin{array}{l}164 \\
936 \\
1996 \\
4566 \\
1809\end{array}$ & $\begin{array}{l}5.29 \\
7.74 \\
10.92 \\
15.00 \\
18.38\end{array}$ & $<0.001$ \\
\hline $\begin{array}{l}\text { No of living persons } \\
\begin{array}{l}1-3 \\
4-6 \\
7 / 7+\end{array}\end{array}$ & $\begin{array}{l}19739 \\
42236 \\
11782\end{array}$ & $\begin{array}{l}26.76 \\
57.26 \\
15.97\end{array}$ & $\begin{array}{l}17104 \\
36975 \\
10207\end{array}$ & $\begin{array}{l}86.65 \\
87.54 \\
86.63\end{array}$ & $\begin{array}{l}2635 \\
5261 \\
1575\end{array}$ & $\begin{array}{l}13.35 \\
12.46 \\
13.37\end{array}$ & 0.001 \\
\hline $\begin{array}{l}\text { Presence of a smoking person } \\
\text { No } \\
\text { Yes }\end{array}$ & $\begin{array}{l}60954 \\
12803 \\
\end{array}$ & \begin{tabular}{|l|}
82.64 \\
17.36 \\
\end{tabular} & $\begin{array}{l}59989 \\
4297 \\
\end{array}$ & \begin{tabular}{|l|}
98.42 \\
33.56 \\
\end{tabular} & $\begin{array}{l}965 \\
8506 \\
\end{array}$ & $\begin{array}{l}1.58 \\
66.44 \\
\end{array}$ & $<0.001$ \\
\hline $\begin{array}{l}\text { Use of smokeless tobacco } \\
\text { Yes } \\
\text { No }\end{array}$ & $\begin{array}{l}15032 \\
58725\end{array}$ & $\begin{array}{l}20.38 \\
79.62\end{array}$ & $\begin{array}{l}12250 \\
52036\end{array}$ & $\begin{array}{l}81.49 \\
88.61\end{array}$ & $\begin{array}{l}2782 \\
6689\end{array}$ & $\begin{array}{l}18.51 \\
11.39\end{array}$ & $<0.001$ \\
\hline $\begin{array}{l}\text { Mass media exposure } \\
\text { Yes } \\
\text { No }\end{array}$ & $\begin{array}{l}45876 \\
27881 \\
\end{array}$ & $\begin{array}{l}62.20 \\
37.80\end{array}$ & $\begin{array}{ll}40 & 044 \\
24 & 242\end{array}$ & $\begin{array}{l}87.29 \\
86.95\end{array}$ & $\begin{array}{l}5832 \\
3639\end{array}$ & $\begin{array}{l}12.71 \\
13.05\end{array}$ & 0.182 \\
\hline $\begin{array}{l}\text { Print media exposure } \\
\text { Yes } \\
\text { No }\end{array}$ & $\begin{array}{ll}27 & 422 \\
46 & 335\end{array}$ & $\begin{array}{l}37.18 \\
62.82\end{array}$ & $\begin{array}{l}23969 \\
40317\end{array}$ & $\begin{array}{l}87.41 \\
87.01\end{array}$ & $\begin{array}{l}3453 \\
6018\end{array}$ & $\begin{array}{l}12.59 \\
12.99\end{array}$ & 0.120 \\
\hline
\end{tabular}




\begin{tabular}{|c|c|c|c|c|c|c|c|}
\hline & \multirow{2}{*}{\multicolumn{2}{|c|}{ Total }} & \multicolumn{4}{|c|}{ Tobacco smoking status } & \multirow[t]{3}{*}{$p$} \\
\hline & & & \multicolumn{2}{|l|}{ no } & \multicolumn{2}{|l|}{ yes } & \\
\hline & $n$ & $\%$ & $n$ & $\%$ & $n$ & $\%$ & \\
\hline $\begin{array}{l}\text { Smoking causes serious illness } \\
\text { Yes } \\
\text { No } \\
\text { Do not know }\end{array}$ & $\begin{array}{l}67970 \\
4501 \\
1286 \\
\end{array}$ & $\begin{array}{l}92.15 \\
6.10 \\
1.74 \\
\end{array}$ & $\begin{array}{l}59352 \\
3825 \\
1109\end{array}$ & $\begin{array}{l}87.32 \\
84.98 \\
86.24\end{array}$ & \begin{tabular}{|l|}
8618 \\
676 \\
177 \\
\end{tabular} & $\begin{array}{l}12.68 \\
15.02 \\
13.76 \\
\end{array}$ & $<0.001$ \\
\hline $\begin{array}{l}\text { Smoking causes strokes } \\
\text { Yes } \\
\text { No } \\
\text { Do not know }\end{array}$ & $\begin{array}{l}49924 \\
13449 \\
10384\end{array}$ & $\begin{array}{l}67.69 \\
18.23 \\
14.08\end{array}$ & $\begin{array}{l}43714 \\
11486 \\
9086\end{array}$ & $\begin{array}{l}87.56 \\
85.40 \\
87.50\end{array}$ & \begin{tabular}{|l|}
6210 \\
1963 \\
1298
\end{tabular} & \begin{tabular}{|l|}
12.44 \\
14.60 \\
12.50
\end{tabular} & $<0.001$ \\
\hline $\begin{array}{l}\text { Smoking causes heart attacks } \\
\text { Yes } \\
\text { No } \\
\text { Do not know } \\
\end{array}$ & $\begin{array}{l}57922 \\
9128 \\
6707 \\
\end{array}$ & $\begin{array}{l}78.53 \\
12.38 \\
9.09 \\
\end{array}$ & $\begin{array}{l}50716 \\
7727 \\
5843 \\
\end{array}$ & $\begin{array}{l}87.56 \\
84.65 \\
87.12 \\
\end{array}$ & \begin{tabular}{|l|}
7206 \\
1401 \\
864 \\
\end{tabular} & \begin{tabular}{|l|}
12.44 \\
15.35 \\
12.88 \\
\end{tabular} & $<0.001$ \\
\hline $\begin{array}{l}\text { Smoking causes lung cancer } \\
\text { Yes } \\
\text { No } \\
\text { Do not know }\end{array}$ & $\begin{array}{l}69098 \\
2626 \\
2033 \\
\end{array}$ & $\begin{array}{l}93.68 \\
3.56 \\
2.76 \\
\end{array}$ & $\begin{array}{l}60389 \\
2178 \\
1719 \\
\end{array}$ & $\begin{array}{l}87.40 \\
82.94 \\
84.55\end{array}$ & $\begin{array}{l}8709 \\
448 \\
314\end{array}$ & $\begin{array}{l}12.60 \\
17.06 \\
15.45\end{array}$ & $<0.001$ \\
\hline $\begin{array}{l}\text { Smoking causes TB } \\
\text { Yes } \\
\text { No } \\
\text { Do not know }\end{array}$ & $\begin{array}{l}68624 \\
3015 \\
2118 \\
\end{array}$ & $\begin{array}{l}93.04 \\
4.09 \\
2.87 \\
\end{array}$ & $\begin{array}{l}59856 \\
2590 \\
1840 \\
\end{array}$ & $\begin{array}{l}87.22 \\
85.90 \\
86.87\end{array}$ & \begin{tabular}{|l|}
8768 \\
425 \\
278 \\
\end{tabular} & \begin{tabular}{|l|}
12.78 \\
14.10 \\
13.13 \\
\end{tabular} & 0.098 \\
\hline Total & 73757 & 100.00 & 64286 & 87.16 & 9471 & 12.84 & \\
\hline
\end{tabular}

Table 3 also represents a bivariate analysis of selected covariates by tobacco smoking status. The prevalence of tobacco smoking was higher among respondents aged $46-60$ years. The smoking rate of tobacco smoking was higher in rural areas. The majority of the respondents were in the north east (22.43\%). This study reveals that male respondents had a higher tobacco smoking prevalence, and the smoking rate were also higher among those who had up to primary education. The utmost prevalence of tobacco smoking was $23.46 \%$, and $22.59 \%$ among respondents who were daily wage/causal labourers, and self-employed, respectively. The smoking rate of tobacco smoking was higher in married $(14.29 \%)$ and other $(16.33 \%)$ religious respondents. The tobacco smoking rate was higher in the poorest families, and $66.44 \%$ of the respondents smoking tobacco informed that their family members were also used to smoking. The tobacco smoking rate was also highest for the respondents who use smokeless tobacco. It is observed that all variables considered in this study, excepting only three variables, namely: mass media, print media, and knowledge about tobacco smok- ing causing TB, were highly significantly $(p<0.001)$ associated with tobacco smoking (see the last column of Table 3 ).

Table 4 presents the odds ratio of different risk factors of tobacco smoking. This study found that the respondents' age had a positive effect on tobacco smoking, and it is statistically significant at $1 \%$ level of significance. Tobacco smoking was 1.965 $(\mathrm{OR}=1.965, \mathrm{Cl}: 1.766-2.166 ; p<0.001), 2.497$ (OR = 2.497, Cl: $2.223-2.805 ; p<0.001$ ) and $2.019(\mathrm{OR}=2.019, \mathrm{Cl}: 1.746-2.334$; $p<0.001)$ times higher in the respondents in age group 31-45 years, $46-60$ years and $60+$ years, respectively, compared to the age group 15-20 years. The region of the respondents had a significant negative effect, except the north east region, on tobacco smoking. The central, east, west and south regions were 0.677 (OR $=0.677$, $\mathrm{Cl}: 0.599-0.766 ; p<0.001), 0.590$ (OR = 0.590, Cl: 0.517-0.674; $p<0.001$ ), 0.345 (OR $=0.345, \mathrm{Cl}: 0.293-0.406 ; p<0.001$ ) and 0.639 (OR $=0.639, \mathrm{Cl}: 0.567-0.721 ; p<0.001)$ times less likely to smoke tobacco than the north region, respectively. But the north east region was 1.451 times $(\mathrm{OR}=1.451, \mathrm{Cl}$ : 1.295-1.625; $p<0.001)$ more likely to smoke tobacco than the north region.

\begin{tabular}{|c|c|c|c|c|c|}
\hline \multirow[t]{2}{*}{ Characteristics } & \multirow[t]{2}{*}{ Coefficient } & \multirow[t]{2}{*}{$p$} & \multirow[t]{2}{*}{ OR } & \multicolumn{2}{|c|}{ 95\% C.I. for OR } \\
\hline & & & & lower & upper \\
\hline $\begin{array}{l}\text { Age group } \\
\begin{array}{l}15-30 \text { years (Ref) } \\
31-45 \text { years } \\
46-60 \text { years } \\
61+\text { years }\end{array}\end{array}$ & $\begin{array}{l}0.671 \\
0.915 \\
0.702 \\
\end{array}$ & $\begin{array}{l}<0.001 \\
<0.001 \\
<0.001\end{array}$ & $\begin{array}{l}1.000 \\
1.956 \\
2.497 \\
2.019 \\
\end{array}$ & $\begin{array}{l}1.766 \\
2.223 \\
1.746 \\
\end{array}$ & $\begin{array}{l}2.166 \\
2.805 \\
2.334 \\
\end{array}$ \\
\hline $\begin{array}{l}\text { Residence } \\
\text { Urban (Ref) } \\
\text { Rural } \\
\end{array}$ & -0.026 & 0.561 & $\begin{array}{l}1.000 \\
0.975 \\
\end{array}$ & 0.893 & 1.063 \\
\hline $\begin{array}{l}\text { Region } \\
\text { North (Ref) } \\
\text { Central } \\
\text { East } \\
\text { North East } \\
\text { West } \\
\text { South }\end{array}$ & $\begin{array}{l}-0.390 \\
-0.528 \\
0.372 \\
-1.065 \\
-0.448\end{array}$ & $\begin{array}{l}<0.001 \\
<0.001 \\
<0.001 \\
<0.001 \\
<0.001\end{array}$ & $\begin{array}{l}1.000 \\
0.677 \\
0.590 \\
1.451 \\
0.345 \\
0.639\end{array}$ & $\begin{array}{l}0.599 \\
0.517 \\
1.295 \\
0.293 \\
0.567\end{array}$ & $\begin{array}{l}0.766 \\
0.674 \\
1.625 \\
0.406 \\
0.721\end{array}$ \\
\hline
\end{tabular}




\begin{tabular}{|c|c|c|c|c|c|}
\hline \multirow[t]{2}{*}{ Characteristics } & \multirow[t]{2}{*}{ Coefficient } & \multirow[t]{2}{*}{$p$} & \multirow[t]{2}{*}{ OR } & \multicolumn{2}{|c|}{ 95\% C.I. for OR } \\
\hline & & & & lower & upper \\
\hline $\begin{array}{l}\text { Gender } \\
\text { Male (Ref) } \\
\text { Female } \\
\end{array}$ & -1.434 & $<0.001$ & $\begin{array}{l}1.000 \\
0.238 \\
\end{array}$ & 0.209 & 0.273 \\
\hline $\begin{array}{l}\text { Education } \\
\text { No education (Ref) } \\
\text { Up to primary } \\
\text { Up to secondary } \\
\text { More than secondary }\end{array}$ & $\begin{array}{l}-0.239 \\
-0.568 \\
-0.786\end{array}$ & $\begin{array}{l}<0.001 \\
<0.001 \\
<0.001\end{array}$ & $\begin{array}{l}1.000 \\
0.787 \\
0.567 \\
0.456\end{array}$ & $\begin{array}{l}0.711 \\
0.510 \\
0.401\end{array}$ & $\begin{array}{l}0.872 \\
0.630 \\
0.517\end{array}$ \\
\hline $\begin{array}{l}\text { Occupation } \\
\text { Govt./Non-Govt. Employee (Ref) } \\
\text { Daily Wage/Casual Labourer } \\
\text { Self-Employed } \\
\text { Homemaker } \\
\text { Student } \\
\text { Others } \\
\end{array}$ & $\begin{array}{l}0.307 \\
0.172 \\
-0.084 \\
-1.074 \\
0.025 \\
\end{array}$ & $\begin{array}{l}<0.001 \\
0.003 \\
0.345 \\
<0.001 \\
0.765 \\
\end{array}$ & $\begin{array}{l}1.000 \\
1.360 \\
1.188 \\
0.919 \\
0.342 \\
1.025 \\
\end{array}$ & $\begin{array}{l}1.203 \\
1.059 \\
0.772 \\
0.277 \\
0.872 \\
\end{array}$ & $\begin{array}{l}1.537 \\
1.333 \\
1.095 \\
0.420 \\
1.205 \\
\end{array}$ \\
\hline $\begin{array}{l}\text { Religion } \\
\text { Hindu (Ref) } \\
\text { Others } \\
\end{array}$ & 0.173 & $<0.001$ & $\begin{array}{l}1.000 \\
1.189 \\
\end{array}$ & 1.089 & 1.297 \\
\hline $\begin{array}{l}\text { Marital status } \\
\text { Not married (Ref) } \\
\text { Currently married } \\
\text { Formerly married } \\
\end{array}$ & $\begin{array}{l}0.934 \\
0.939 \\
\end{array}$ & $\begin{array}{l}<0.001 \\
<0.001 \\
\end{array}$ & $\begin{array}{l}1.000 \\
2.544 \\
2.557 \\
\end{array}$ & $\begin{array}{l}2.249 \\
2.082 \\
\end{array}$ & $\begin{array}{l}2.877 \\
3.139 \\
\end{array}$ \\
\hline $\begin{array}{l}\text { Wealth index } \\
\text { Richest (Ref) } \\
\text { Rich } \\
\text { Middle } \\
\text { Poor } \\
\text { Poorest } \\
\end{array}$ & $\begin{array}{l}0.226 \\
0.373 \\
0.433 \\
0.629 \\
\end{array}$ & $\begin{array}{l}0.019 \\
<0.001 \\
<0.001 \\
<0.001\end{array}$ & $\begin{array}{l}1.000 \\
1.254 \\
1.452 \\
1.542 \\
1.876 \\
\end{array}$ & $\begin{array}{l}1.038 \\
1.213 \\
1.289 \\
1.532 \\
\end{array}$ & $\begin{array}{l}1.514 \\
1.738 \\
1.845 \\
2.296 \\
\end{array}$ \\
\hline $\begin{array}{l}\text { No of living persons } \\
1-3 \text { (Ref) } \\
4-6 \\
7 / 7+ \\
\end{array}$ & $\begin{array}{l}-0.368 \\
-0.859 \\
\end{array}$ & $\begin{array}{l}<0.001 \\
<0.001\end{array}$ & $\begin{array}{l}1.000 \\
0.692 \\
0.423 \\
\end{array}$ & $\begin{array}{l}0.634 \\
0.379 \\
\end{array}$ & $\begin{array}{l}0.755 \\
0.473 \\
\end{array}$ \\
\hline $\begin{array}{l}\text { Presence of smoking person } \\
\text { Yes (Ref) } \\
\text { No }\end{array}$ & -4.628 & $<0.001$ & $\begin{array}{l}1.000 \\
0.010 \\
\end{array}$ & 0.009 & 0.011 \\
\hline $\begin{array}{l}\text { Use of smokeless tobacco } \\
\text { Yes (Ref) } \\
\text { No }\end{array}$ & 0.085 & 0.040 & $\begin{array}{l}1.000 \\
1.088 \\
\end{array}$ & 1.004 & 1.180 \\
\hline $\begin{array}{l}\text { Mass media exposure } \\
\text { Yes (Ref) } \\
\text { No }\end{array}$ & 0.059 & 0.175 & $\begin{array}{l}1.000 \\
1.060\end{array}$ & 0.974 & 1.154 \\
\hline $\begin{array}{l}\text { Print media exposure } \\
\text { Yes (Ref) } \\
\text { No } \\
\end{array}$ & -0.006 & 0.891 & $\begin{array}{l}1.000 \\
0.994 \\
\end{array}$ & 0.914 & 1.081 \\
\hline $\begin{array}{l}\text { Smoking causes serious illness } \\
\text { Yes (Ref) } \\
\text { No } \\
\text { Do not know }\end{array}$ & $\begin{array}{l}0.001 \\
-0.210 \\
\end{array}$ & $\begin{array}{l}0.996 \\
0.143 \\
\end{array}$ & $\begin{array}{l}1.000 \\
1.000 \\
0.811 \\
\end{array}$ & $\begin{array}{l}0.862 \\
0.613 \\
\end{array}$ & $\begin{array}{l}1.160 \\
1.073 \\
\end{array}$ \\
\hline $\begin{array}{l}\text { Smoking causes strokes } \\
\text { Yes (Ref) } \\
\text { No } \\
\text { Do not know }\end{array}$ & $\begin{array}{l}0.135 \\
0.123 \\
\end{array}$ & $\begin{array}{l}0.017 \\
0.076 \\
\end{array}$ & $\begin{array}{l}1.000 \\
1.145 \\
1.131 \\
\end{array}$ & $\begin{array}{l}1.025 \\
0.987 \\
\end{array}$ & $\begin{array}{l}1.279 \\
1.295 \\
\end{array}$ \\
\hline $\begin{array}{l}\text { Smoking causes heart attacks } \\
\text { Yes (Ref) } \\
\text { No } \\
\text { Do not know }\end{array}$ & $\begin{array}{l}0.065 \\
-0.169 \\
\end{array}$ & $\begin{array}{l}0.331 \\
0.049 \\
\end{array}$ & $\begin{array}{l}1.000 \\
1.067 \\
0.844 \\
\end{array}$ & $\begin{array}{l}0.936 \\
0.713 \\
\end{array}$ & $\begin{array}{l}1.218 \\
0.999 \\
\end{array}$ \\
\hline $\begin{array}{l}\text { Smoking causes lung cancer } \\
\text { Yes (Ref) } \\
\text { No } \\
\text { Do not know }\end{array}$ & $\begin{array}{l}0.241 \\
0.276 \\
\end{array}$ & $\begin{array}{l}0.038 \\
0.037 \\
\end{array}$ & $\begin{array}{l}1.000 \\
1.273 \\
1.318 \\
\end{array}$ & $\begin{array}{l}1.014 \\
1.017 \\
\end{array}$ & $\begin{array}{l}1.599 \\
1.707 \\
\end{array}$ \\
\hline $\begin{array}{l}\text { Smoking causes TB } \\
\text { Yes (Ref) } \\
\text { No } \\
\text { Do not know }\end{array}$ & $\begin{array}{l}-0.082 \\
-0.198\end{array}$ & $\begin{array}{l}0.448 \\
0.125\end{array}$ & $\begin{array}{l}1.000 \\
0.922 \\
0.820\end{array}$ & $\begin{array}{l}0.746 \\
0.636\end{array}$ & $\begin{array}{l}1.138 \\
1.057\end{array}$ \\
\hline
\end{tabular}

OR - odds ratio. 
From the results, it is clear that the gender of respondents has a negative effect on tobacco smoking. The possibility of tobacco smoking for females was a $0.238(\mathrm{OR}=0.238, \mathrm{Cl}$ : $0.209-$ $-0.273 ; p<0.001$ ) times lower risk compared to males. It is observed that there is an inverse relationship between education status and tobacco smoking. That means increasing education status from primary to higher; the amount of tobacco smoking is decreased, because an educated person is more conscious of their health. The possibility of tobacco smoking of up to primary, up to secondary, and more than secondary educated respondents was $0.787(\mathrm{OR}=0.787, \mathrm{Cl}: 0.711-0.872 ; p<0.001)$, 0.567 (OR $=0.567, \mathrm{Cl}: 0.510-0.630 ; p<0.001)$, and $0.456(\mathrm{OR}=$ $0.456, \mathrm{Cl}: 0.401-0.517 ; p<0.001$ ) times less likely compared to uneducated respondents, respectively. These results of occupation showed that daily wage/casual labourer and self-employed had a significant positive impact, and student had a significant negative impact on tobacco smoking. The possibility of tobacco smoking for a daily wage/casual labourer and self-employed was 1.360 (OR = 1.360, Cl: 1.203-1.537; $p<0.001)$ and 1.188 (OR $=1.188, \mathrm{Cl}: 1.059-1.333 ; p=0.003)$ times higher compared to a government/non-government employee. On the other hand, the respondents who were students had 0.342 (OR $=0.342$, $\mathrm{Cl}: 0.277-0.420 ; p<0.001)$ times lower tobacco smoking than government/non-government employees. The likelihood of tobacco smoking in others families had 1.189 (OR $=1.189$, Cl: $1.089-1.297 ; p<0.001)$ times higher compared to a Hindu family. The marital status of respondents had a positive significant effect on tobacco smoking. The chance of tobacco smoking for currently married and formerly married was 2.544 (OR $=2.544, \mathrm{Cl}: 2.249-2.877 ; p<0.001)$ and $2.557(\mathrm{OR}=2.557, \mathrm{Cl}$ : 2.082-3.139; $p<0.001)$ times more likely compared to unmarried respondents. Wealth index had a significant positive effect on tobacco smoking. The chance of tobacco smoking for rich, middle, poor and poorest families was $1.254(\mathrm{OR}=1.254$, Cl: 1.038-1.514; $p=0.019), 1.452$ (OR = 1.452, Cl: 1.213-1.738; $p<0.001), 1.542$ (OR $=1.542, \mathrm{Cl}: 1.289-1.845 ; p<0.001$ ) and $1.876(\mathrm{OR}=1.876, \mathrm{Cl}: 1.532-2.296 ; p<0.001)$ times, respectively, more likely compared to the richest families. These results of the number of living person indicate that it has a significant negative impact on tobacco smoking. The likelihood of tobacco smoking for $4-6$ and $7+$ number of living person was 0.692 (OR $=0.692, \mathrm{Cl}: 0.634-0.755 ; p<0.001)$, and 0.423 (OR $=0.423$, Cl: $0.379-0.473 ; p<0.001)$ times lower than $1-3$ number of living persons. Families having a smoking person had a significant negative effect on tobacco smoking. The chance of tobacco smoking for absence of smoking person was 0.010 times $(\mathrm{OR}=$ $0.010, \mathrm{Cl}: 0.009-0.011 ; p<0.001$ ) less likely compared to the presence of a smoking person. Use of smokeless tobacco also has a significant positive effect on tobacco smoking. That means tobacco smoking was $1.088(\mathrm{OR}=1.088, \mathrm{Cl}: 1.004-1.118$; $p=0.040$ ) times higher than the respondents who used smokeless tobacco. Respondents having no knowledge that smoking causes stroked had a significant positive effect on tobacco smoking. The chance of tobacco smoking for respondents having no knowledge that smoking causes strokes were 1.145 times (OR $=1.145, \mathrm{Cl}: 1.025-1.279 ; p=0.017$ ) higher than those having knowledge that smoking causes strokes. Knowledge of smoking's association with lung cancer has a significant positive effect on tobacco smoking. The likelihood of tobacco smoking for no knowledge of smoking's association with lung cancer was 1.273 times (OR $=1.273, \mathrm{Cl}: 1.014-1.599 ; p=0.038)$ higher than that having knowledge of smoking's association with lung cancer.

\section{Discussion}

In this study, nationwide large scale GATS-2017 India data were used, and it provide a sufficient study on tobacco smoking behaviour among adults. To analyze the prevalence and risk factors of tobacco smoking, different statistical techniques and models were developed and carried out. Therefore, there is no scope for comparing the developed statistical techniques and models in the present study, but it is important to compare this study with other studies. This study found that the national prevalence of tobacco smoking was $12.84 \%$ in India, with $25.01 \%$ for males and $2.63 \%$ for females. This finding is much higher than national studies from Ethiopia [2] and Ghana [18], but lower than national studies from Madagascar [19] and Nepal [20]. The social acceptance of tobacco consumption is also high in Nepal [20]. This study revealed variations in tobacco smoking all over India's regional states, and similar results were found in Ethiopia [2]. The highest prevalence was found in the north east region, and this result is not supported by the previous surveys' results in India [7]. Tobacco smoking in India is higher among males than females. In this study the female tobacco smoking rate is $2.63 \%$ in India, whereas this rate is higher than Pakistan (1.8\%) [6]. In India tobacco smoking is higher among up to primary educated, married, poorest and casual labourer. This result is supported by Singh and Ladusingh [7]. The odds of tobacco smoking was found to be more likely among the middle and older age groups. Specifically, those who were in the age group 46-60 years were more likely to smoke tobacco compared to those in the 15 to 30 years age group, and this is consistent with a previous study of India [7]. This finding is also more similar to a study from Nepal [20], a town surveys in south-central Ethiopia [21], Ghana [18], Brazil [22] and Madagascar [19]. One important fact is that older adults have had a longer time experience to smoke tobacco, and have developed the bad habit of tobacco smoking [23]. A person who started tobacco smoking in early life has been found to have less chance of giving up tobacco smoking in future life [24]. This study showed that older adults (65+ years) were more likely to ignore tobacco smoking, and these findings are supported by Van Loon et al. [25].

Tobacco smoking in India varies significantly by national region. The risk of tobacco smoking among adults is more likely in the north east region compared to the north region. This finding is consistent with a previous study on GTAS-India 2009 data $[7,8]$. Males are more likely to smoke tobacco as compared to females. Several studies in Africa have shown that tobacco smoking is more prevalent in males [23, 26-28]. Females who are in the habit of tobacco smoking face humiliation and discrimination by their own community in India. Females are also more socially restricted than their male counterparts [23]. This is supported by Reda et al. [23]. Education was one of the most important factors of tobacco smoking among adults in India. Adults with no education were at higher risk, and more than higher educated adults were at a lower risk of tobacco smoking in India. This finding is supported by the previous study in 2009 using GTAS-India data [7], and survey analysis in the Butajira town of Ethiopia [21]. This study found that occupation type was associated with tobacco smoking. Daily wage/casual labourer were associated with higher odds of tobacco smoking as compared to adult government/non-government employees. Lakew and Haile found that professional working adults were associated with lower odds of tobacco smoking in Ethiopia [2]. The possible justification could be that ethics demanded for professional workers might prevent them from tobacco use [2]. A study in Nepal found that adults in labour-intensive occupations had increased odds of using tobacco smoking as compared to government/non-government (professional) jobs [20]. In Madagascar, occupation type was also significantly associated with tobacco smoking [19]. The odds of tobacco smoking among currently married and formerly married adults were around $54 \%$ and $56 \%$ higher as compared to unmarried adults. This result is supported by Lakew and Haile [2]. Being among the poorest was significantly associated with a higher risk of tobacco smoking among adults in India [2]. The odds of tobacco smoking increased when the respondent wealth index was decreasing from the richest to the poorest. This finding is supported by Singh and Ladusingh [7] and Nketiah-Amponsah et al. [29]. The number of living persons in a family is a significant associated 
factor of tobacco smoking, and the odds of tobacco smoking were decreased if the number of living persons was increased. Knowledge about the health hazards of tobacco smoking is important for finding the important determinants. The health risks of tobacco smoking are sometimes not understood by tobacco smokers [30]. Most of the adults knew that strokes and lung cancer are the most common diseases induced by smoking. There are several others non-communicable diseases which are caused by tobacco smoking. The risk of tobacco smoking was higher among adults who were not aware that tobacco smoking causes lung cancer and strokes in India. Singh and Ladusingh do not support this finding, and they obtained the reverse results [7]. The GATS-India 2017 report shows that knowledge about the health hazards of tobacco smoking is moderately high in India, and similar results were found in GATS-India 2009 reports. So there is a need to raise awareness about the effects of tobacco smoking.

As this study utilized a nationally representative sample from India, the results can be generalized to the target population ( $\geq 15$-year-old population in India). Moreover, due to the utilization of standard and valid tools for data collection by GATS, the probability of the existence of measurement error is less in this study in comparison to other single cross-sectional studies conducted in India.

\section{Limitations and recommendations of the study}

However, this study has some potential limitations. Although a standardized procedure and questionnaire were utilized in this study, the limitations of this study cannot be ignored. The cross-sectional design of the study could not enable us to establish correlations between variables. Also, the questionnaire was controlled by interviewers, so there was a risk of interviewer bias. Though multistage random sampling was used for the selection of respondents, it could be subject to some selection bias if the interviewer did not follow sampling instructions properly. The survey was limited to household population in 32 states, and findings cannot be generalized to segments that were excluded, as discussed in the methods section. The prevalence and risk factors of smoking were measured at one point in time. Therefore, it is not possible to determine the direction of causal relationships between smoking and some factors, like income.

These findings support the need for strengthening the tobacco control programme in India. In detail, this study recommends the following:

- There was a significant decrease in tobacco smoking among adults compared to a previous study in India, and the tobacco control programme needs to focus on its strategies and continue these strategies. In India there exist rules that ban tobacco smoking in public places. To reduce tobacco smoking among adults the advertisement about the bad impact of tobacco products in electronic media is essential. Under such situations, only putting health warnings on cigarette packaging is not enough to control the tobacco smoking epidemic in the country.

- The tobacco smoking rate was higher among males than females at the current time. So to reduce the number of male smokers there needs to more awareness about the harmful effects of tobacco smoking. Female tobacco smoking is at a low level, and continued efforts are needed to keep it at low levels.

- The poorer and uneducated are at high risk of tobacco smoking. So tobacco control campaigns should demonstrate a motivation to quit. An increase in price and taxation on tobacco products can decrease its consumption. Capitalizing in education would have additional benefits for tobacco control because higher education makes a person more conscious about health.

- Formerly married people, and families having a smoking person are also at high risk of tobacco smoking. A formerly married person feels lonely and affected by depression, so they need more awareness and entertainment. Most people start smoking in their teen years. They might start because their parents or other family members smoke. If the family member is educated and conscious about their health and future, then it will help to reduce tobacco smoking.

- Systematic surveys on the prevalence and risk factors of tobacco smoking in the general population should be carried out at regular time intervals. Otherwise, the progress of the tobacco smoking control programme will be unable to control the smoking epidemic.

\section{Conclusions}

This study used nationally representative data to discover the prevalence and factors that affect tobacco smoking intensity among adults in India. The overall prevalence of tobacco smoking seems relatively high in India. There are some regional states, specifically Arunachal Pradesh, Manipur, Meghalaya, Mizoram, and Tripura, that need special attention because of their higher prevalence (more than 20\%) of tobacco smoking. All factors had a statistically significant association with tobacco smoking except the mass media exposure, print media exposure, and knowledge that tobacco smoking causes TB variables. Age, national region, gender, education, occupation, religion, marital status, wealth index, number of living persons, presence of smoking persons, use of smokeless tobacco, and knowledge that smoking causes strokes and lung cancer had a statistically significant impact on tobacco smoking. As a result, these factors should be considered when pointing out specific public health interventions to reduce tobacco smoking in India. High prevalence regions of tobacco smoking need extraordinary intervention. This can often be endorsed among people with less knowledge and awareness of the bad impact of tobacco smoking.

Acknowledgements. The authors acknowledge Measure GATS for granting the data for this study. The authors thankfully acknowledge the contribution of the Statistics Discipline, Science, Engineering and Technology School, Khulna University, Khulna-9208, Bangladesh. Thanks to Molla Azizur Rahman, English Discipline, Khulna University, Bangladesh, and also thanks to the editor and reviewers for their comments and criticism to improve this paper.

Source of funding: This work was funded from the authors' own resources.

Conflicts of interest: The authors declare no conflicts of interest.

\section{References}

1. Mishra GA, Pimple SA, Shastri SS. An overview of the tobacco problem in India. Indian J Med Paediatr Oncol 2012; 33: $139-145$.

2. Lakew Y, Haile D. Tobacco use and associated factors among adults in Ethiopia: further analysis of the 2011 Ethiopian Demographic and Health Survey. BMC Public Health 2015; 15: 487, doi: 10.1186/s12889-015-1820-4.

3. Doku D, Koivusilta L, Raisamo S, et al. Do socioeconomic differences in tobacco use exist also in developing countries? A study of Ghanaian adolescents. BMC Public Health 2010; 10: 758, doi: 10.1186/1471-2458-10-758. 
4. Doku D, Raisamo S, Wiium N. The role of tobacco promoting and restraining factors in smoking intentions among Ghanaian youth. $B M C$ Public Health 2012; 12: 662, doi: 10.1186/1471-2458-12-662.

5. Sreeramareddy CT, Pradhan PM, Sin S. Prevalence, distribution, and social determinants of tobacco use in 30 sub-Saharan African countries. BMC Med 2014; 12: 243, doi: 10.1186/s12916-014-0243-x.

6. Gilani SI, Leon DA. Prevalence and sociodemographic determinants of tobacco use among adults in Pakistan: findings of a nationwide survey conducted in 2012. Popul Health Metr 2013; 11(1): 16, doi: 10.1186/1478-7954-11-16.

7. Singh A, Ladusingh L. Prevalence and determinants of tobacco use in India: evidence from recent global adult tobacco survey data. PLOS ONE 2014; 9(12): e114073, doi: 10.1371/journal.pone.0114073.

8. Rani M, Bonu S, Jha P, et al. Tobacco use in India: prevalence and predictors of smoking and chewing in a national cross sectional household survey. Tob Control 2003; 12: e4-e4.

9. Owolabi EO, Goon DT, Adeniyi OV, et al. Prevalence and factors associated with tobacco use among adults attending selected healthcare facilities in Buffalo City Metropolitan Municipality, South Africa. SAFP 2017; 59: 201-207.

10. Jha P, Jacob B, Gajalakshmi V, et al. A nationally representative case-control study of smoking and death in India. N Engl J Med 2008; 358: 1137-1147.

11. World Health Organization. WHO report on the global tobacco epidemic, 2008. The MPOWER package. Geneva: WHO; 2008.

12. Giovino GA, Mirza SA, Samet JM, et al. GATS Collaborative Group. Tobacco use in 3 billion individuals from 16 countries: an analysis of nationally representative cross-sectional household surveys. Lancet 2012; 380: 668-679.

13. Jha P. Avoidable deaths from smoking: a global perspective. Public Health Rev 2011; 33: 569-600.

14. Ekpu VU, Brown AK. The economic impact of smoking and of reducing smoking prevalence: review of evidence. Tob Use Insights 2015; 8: 1-35, doi: 10.4137/TUI.S15628.

15. Chan YH. Biostatistics 103: Qualitative data-tests of independence. Singapore Med J 2003; 44: 498-503.

16. Rudatsikira E, Abdo A, Muula AS. Prevalence and determinants of adolescent tobacco smoking in Addis Ababa, Ethiopia. BMC Public Health 2007; 7: 176, doi: 10.1186/1471-2458-7-176.

17. Filippidis FT, Vardavas $\mathrm{Cl}$, Loukopoulou A, et al. Prevalence and determinants of tobacco use among adults in Greece: 4 year trends. Eur J Public Health 2012; 23: 772-776.

18. Doku D, Darteh EKM, Kumi-Kyereme A. Socioeconomic inequalities in cigarette smoking among men: evidence from the 2003 and 2008 Ghana demographic and health surveys. Arch Public Health 2013; 71(1): 9, doi: 10.1186/0778-7367-71-9.

19. Mamudu HM, John RM, Veeranki SP, et al. The odd man out in Sub-Saharan Africa: understanding the tobacco use prevalence in Madagascar. BMC Public Health 2013; 13: 856, doi: 10.1186/1471-2458-13-856.

20. Khanal V, Adhikari M, Karki S. Social determinants of tobacco consumption among Nepalese men: findings from Nepal Demographic and Health Survey 2011. Harm Reduct J 2013; 10: 40, doi: 10.1186/1477-7517-10-40.

21. Schoenmaker N, Hermanides J, Davey G. Prevalence and predictors of smoking in Butajira town, Ethiopia. EJHD 2005; $19: 182-187$.

22. Peixoto SV, Firmo JOA, Lima-Costa MF. Factors associated to smoking habit among older adults (The Bambuí Health and Aging Study). Rev Saude Publica 2005; 39: 746-753.

23. Reda AA, Moges A, Yazew B, et al. Determinants of cigarette smoking among school adolescents in eastern Ethiopia: a cross-sectional study. Harm Reduct J 2012; 9: 39, doi: 10.1186/1477-7517-9-39.

24. Caponnetto P, Polosa R. Common predictors of smoking cessation in clinical practice. Respir Med 2008 102(8): 1182-1192, doi: 10.1016/j.rmed.2008.02.017.

25. Van Loon AJM, Tijhuis M, Surtees PG, et al. Determinants of smoking status: cross-sectional data on smoking initiation and cessation. Eur J Public Health 2005; 15: 256-261.

26. Hymowitz N, Cummings KM, Hyland A, et al. Predictors of smoking cessation in a cohort of adult smokers followed for five years. Tob Control 1997; 6: S57-S62.

27. Rudatsikira E, Dondog J, Siziya S, et al. Prevalence and determinants of adolescent cigarette smoking in Mongolia. Singapore Med J 2008; 49: 57-62.

28. Steyn K, Bradshaw D, Norman R, et al. Tobacco use in South Africans during 1998: the first demographic and health survey. J Cardiovasc Risk 2002; 9: 161-170.

29. Nketiah-Amponsah E, Afful-Mensah G, Ampaw S. Determinants of cigarette smoking and smoking intensity among adult males in Ghana. BMC Public Health 2018; 18(1): 941, doi: 10.1186/s12889-018-5872-0.

30. World Health Organization. WHO report on the global tobacco epidemic 2011. Warning about the dangers of tobacco. Geneva: WHO; 2011.

Tables: 4

Figures: 4

References: 30

Received: 6.04.2019

Reviewed: 5.05.2019

Accepted: 14.05.2019

Address for correspondence:

Benojir Ahammed, M.Sc., Assist. Prof.

Statistics Discipline

Khulna University

Khulna-9208

Bangladesh

Tel.: +880 1516768643

E-mail: benojirstat@gmail.com 